This is an electronic reprint of the original article. This reprint may differ from the original in pagination and typographic detail.

\author{
Author(s): Miettinen, Arttu; Ojala, Antti; Wikström, Lisa; Joffe, Roberts; Madsen, Bo; Nättinen, \\ Kalle; Kataja, Markku
}

Title: $\quad$ Non-destructive automatic determination of aspect ratio and cross-sectional properties of fibres

Year: $\quad 2015$

Version:

Please cite the original version:

Miettinen, A., Ojala, A., Wikström, L., Joffe, R., Madsen, B., Nättinen, K., \& Kataja, M. (2015). Non-destructive automatic determination of aspect ratio and cross-sectional properties of fibres. Composites Part A: Applied Science and Manufacturing, 77(October), 188-194. https://doi.org/10.1016/j.compositesa.2015.07.005

All material supplied via JYX is protected by copyright and other intellectual property rights, and duplication or sale of all or part of any of the repository collections is not permitted, except that material may be duplicated by you for your research use or educational purposes in electronic or print form. You must obtain permission for any other use. Electronic or print copies may not be offered, whether for sale or otherwise to anyone who is not an authorised user. 


\title{
Non-destructive automatic determination of aspect ratio and cross-sectional properties of fibres
}

\author{
Arttu Miettinen*1, Antti Ojala ${ }^{\dagger 2}$, Lisa Wikström² ${ }^{\ddagger 2}$ Roberts Joffe $^{\S 3}$, Bo Madsen ${ }^{\uparrow 4}$, Kalle Nättinen ${ }^{\| 5}$, and \\ Markku Kataja**1 \\ ${ }^{1}$ University of Jyvaskyla, Department of Physics, P.O. Box 35 (YFL) FI-40014 Jyvaskyla, Finland \\ ${ }^{2}$ VTT Technical Research Centre of Finland, Sinitaival 6, P.O. Box 1300, FI-33101 Tampere, Finland \\ ${ }^{3}$ Luleå University of Technology, Composite Centre Sweden, SE-97187 Luleå, Sweden \\ ${ }^{4}$ Technical University of Denmark, Department of Wind Energy, Risø Campus, P.O. Box 49, DK-4000 \\ Roskilde, Denmark \\ ${ }^{5}$ Bemis Flexible Packaging Europe, Teollisuustie 1, FI-37601 Valkeakoski, Finland
}

Composites: Part A 77 (2015) 188-194, doi: 10.1016/j.compositesa.2015.07.005

\section{Abstract}

A novel method for computerized estimation of the aspect ratio distribution and various cross-sectional geometrical properties of fibres in short-fibre reinforced composites is proposed. The method, based on X-ray microcomputed tomography, is non-destructive and does not require user intervention. Based on results on specially fabricated model material, the accuracy and precision of the method seems adequate. The method is applied in analysing a manufacturing process of wood fibre reinforced thermoplastic composite. The results indicate a significant decrease of the aspect ratio of fibres during the processing steps. Finally, the feasibility of the method is assessed by estimating parameters of a micromechanical model for flax fibre composites and comparing the results with those from tensile tests.

\footnotetext{
*arttu.miettinen@phys.jyu.fi

†antti.ojala@vtt.fi

${ }^{\ddagger}$ lisa.wikstrom@vtt.fi

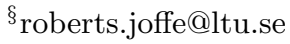

『boma@dtu.dk

"knattinen@bemis.com

**markku.kataja@jyu.fi
}

\section{Introduction}

Within micromechanical approach to estimating composite material performance, quantities describing the microscopic or mesoscopic structure of a composite are combined with mechanical properties of the constituents in order to estimate the macroscopic properties of the material [1-4]. For short-fibre reinforced composite materials the relevant statistical quantities describing the microstructure include, e.g., diameter, length and orientation distributions of fibres, and volume fractions of the constituents. The microstructure of such materials is formed during the manufacturing processes that typically include, e.g., pelletization, compounding, extrusion and moulding, each of which affect the geometry and distribution of the precursors [5]. Information on the microstructure of the material $[6,7]$ evolving through the different processing stages may be used to optimize the processing chain and the properties of the final product through selection of proper processing methods $[8,9]$ and their parameters [10].

Traditionally, composite material microstructure is studied by dissolving the matrix and studying the residual fibres by optical- or electron microscopy. Alternatively, polished cross-sections of the composite may be used. The methods are accurate, but time-consuming and destructive. Additionally, the applied chemical or mechanical treatments involved in the analysis process may change 
the structure of the fibres and, furthermore, in the dissolution process information about orientation and dispersion of the fibres is lost.

$\mathrm{X}$-ray microtomography $(\mathrm{X}-\mu \mathrm{CT})$ is a non-destructive method to characterize the structure of heterogeneous materials. The method is based on computationally reconstructing the three-dimensional structure of the sample from a number of two-dimensional X-ray attenuation images taken from different directions [11]. Contrast in such a tomographic image typically originates from local X-ray attenuation coefficient that correlates with local density of the material sample. The spatial resolution available $(\sim$ $1 \mu \mathrm{m})$ is adequate for distinguishing the different phases, e.g., fibres, matrix and void in many composites (see Figure 1b). Traditional image processing algorithms [12] may be used to segment the different phases and thereby to produce, for further processing, a three-dimensional binarized image showing, e.g., the fibre phase only.

Several methods for estimating fibre properties from a binary image have been recently proposed. Majority of the methods are based on separating individual fibres from each other. Methods based on analysing the medial axis of fibres [13-17] are often applicable to solid fibres, whereas methods based on recognition of lumen [18-21] apply to hollow fibres. In the case of natural fibres, processing may break and crush the fibres such that their shape becomes highly irregular (see Figure 1b). The fibres may thus be partially hollow and partially solid, hindering the use of above methods. Algorithms introduced in [22-24] are based on analysis of the local orientation of fibres in order to separate them from each other, and thus seem to be better suited for fibres of irregular shape.

Recently, we introduced an automated method to evaluate the fibre length distribution of short-fibre composites from a binarized three-dimensional tomographic image of material sample $[25,26]$. The method does not require identification of individual fibres and includes an algorithm for correcting bias caused by finite image size. In addition, transverse dimensions of fibres could be estimated based on a specific scaling assumption for fibre volume. In this work we relax the need for such an assumption and introduce an improved method to determine the statistical distribution of various geometrical properties of fibre cross-section as well as of fibre length. In particular, the present method can be used to correlate fibre length and cross-sectional properties such that, e.g., the aspect ratio distribution can be obtained. The technique is validated using a model composite with known structure.

The method is then applied in assessing the effects of various manufacturing unit processes on the aspect ratio and on the cross-sectional shape of wood-fibres used as reinforcement in a biodegradable composite material. Finally, we apply the method in finding the necessary parameters for a micromechanical model for estimating Young's modulus of flax fibre composites, the results of which are compared with those from tensile tests.

\section{Estimation of microstructural prop- erties}

Let us denote a three-dimensional binarized tomographic image of a short-fibre reinforced composite material sample by $I\left(x_{1}, x_{2}, x_{3}\right)$. The numeric value of each voxel, located at position $\vec{x}=\left(x_{1}, x_{2}, x_{3}\right)$, indicates whether or not that particular point is inside the fibre phase. In order to collect the necessary experimental data for estimating the statistical distribution of various structural descriptors of the fibre phase, the image $I$ is sampled uniformly at random locations such that a statistically significant number of points $\vec{x}_{n}, n=1, \ldots, N$ located inside the fibre phase is found. The local orientation of a fibre in the vicinity of point $\vec{x}_{n}$ can be found using the structure tensor defined by $[27]$

$$
S_{i j}\left(\vec{x}_{n}\right)=\left(G_{\sigma} *\left(I_{i} I_{j}\right)\right)\left(\vec{x}_{n}\right),
$$

where $G_{\sigma}$ is a Gaussian function with zero mean and standard deviation $\sigma$ in all directions, and $*$ denotes convolution. The quantities $I_{i}$ are the partial derivatives of $I$, and can be approximated by [27]

$$
I_{i}\left(\vec{x}_{n}\right)=\frac{\partial I\left(\vec{x}_{n}\right)}{\partial x_{i}} \approx\left(\frac{\partial G_{\sigma}}{\partial x_{i}} * I\right)\left(\vec{x}_{n}\right) .
$$

The eigenvector $\hat{s}_{n}$ of $S_{i j}\left(\vec{x}_{n}\right)$ corresponding to the smallest eigenvalue defines the local fibre orientation at $\vec{x}_{n}$.

Next, a slice is extracted around $\vec{x}_{n}$ such that its normal direction is parallel with $\hat{s}_{n}$ (see Figure 1). The fibre cross-section is extracted from the slice and its area $A_{n}$ is calculated. The cross-sectional dimensions of fibres with non-circular cross-section are characterized here using the lengths of the projections of the cross-section in its two principal directions [28]. For each cross-section we thereby find the principal dimensions $d_{1 n}$ and $d_{2 n}$, with 
convention $d_{1 n} \geq d_{2 n}$. The length of the fibre containing $\vec{x}_{n}$ can be estimated using the constrained path transformation $I(\vec{x}) \rightarrow T(\vec{x})$, where $T$ is an image where the value of each voxel is the length of the longest regular path passing through the corresponding voxel in $I$, and contained entirely inside the fibre phase. The degree of regularity of the path is controlled by a set of topological constraints specific for the algorithm $[25,29]$. The length $L_{n}$ of the fibre at $\vec{x}_{n}$ is then defined as the mode of voxel values in $T$ over the cross-section. Finally, two aspect ratios corresponding to the two principal dimensions $d_{i n}$ can be defined as $r_{i n}=L_{n} / d_{i n}, i=1,2$.

Given the data set $\left\{\hat{s}_{n}, A_{n}, d_{1 n}, d_{2 n}, L_{n}, r_{1 n}, \ldots\right\}, n=$ $1, \ldots, N$, estimates for various multivariate distributions for the structural descriptors can be generated by a straightforward statistical binning. The probability that a randomly selected point in the sample is inside a fibre is proportional to the volume of the fibre. Thus, denoting by $\left\{\xi_{1}, \xi_{2}, \ldots, \xi_{K}\right\}$ any subset of the descriptors (random variables) $\left\{\hat{s}, A, d_{1}, d_{2}, L, r_{1}, \ldots\right\}$ for which the data is measured, the resulting normalized multivariate distributions are of a general type

$$
f\left(\xi_{1}, \xi_{2}, \ldots, \xi_{K}\right)=\frac{1}{V_{f}} \frac{\partial^{K} V_{f}}{\partial \xi_{1} \partial \xi_{2} \ldots \partial \xi_{K}},
$$

i.e., $\xi_{k}$-distributions of fibre phase volume $V_{f}$.

\section{Test case with a model composite}

In order to substantiate the method, a model composite was made from hollow and solid fibres and molding rubber. Steel capillary tube $(0.5 \mathrm{~mm}$ outer diameter and $0.3 \mathrm{~mm}$ inner diameter) was used as hollow fibres, while copper wire $(0.35 \mathrm{~mm}$ diameter $)$ was taken as solid fibres. The tube and the wire were cut into pieces of predetermined lengths between $2 \mathrm{~mm}$ and $20 \mathrm{~mm}$. Predetermined amounts of both types of fibres were mixed with liquid state rubber (Silcoflex HE) such that the mass fraction of fibres was 0.18. The mix was then poured into a cylindrical mold and let to set and solidify. X-ray micromographic image of the material was taken using SkyScan 1172 microtomograph with $17 \mu \mathrm{m}$ pixel size (Bruker-microCT, Belgium), ensuring that all the fibres were visible in the image.

The fibres and the rubber were segmented from the image by simple thresholding so as to produce an image showing only the fibres (see Figure 2). Different types of fibres were identified based on the topological properties of fibre cross-sections such that the simply connected and non-simply connected fibre cross-sections were attributed to solid and hollow fibres, respectively. The aspect ratio distributions were then determined separately for solid and hollow fibres as described in Section 2, based on 50000 cross-sectional samples.

To obtain a reference data set, the rubber matrix and the fibres were separated manually. The fibres were further divided in hollow and solid ones (steel capillary and copper wire) and spread onto a white background cardboard. Photographs of both fractions were taken with $52 \mu \mathrm{m}$ pixel size and calibrated using a length standard. The lengths of all the fibres in the images were then determined manually using the ImageJ software. The lengths and the known cross-sectional area were used to determine reference distributions (i.e. aspect ratio distributions of fibre volume) for the $\mathrm{X}-\mu \mathrm{CT}$ based estimation. A total of 193 hollow fibres and 203 solid ones were measured.

The aspect ratio distributions for both types of fibres are shown in Figure 3, together with the reference distributions. Statistical characteristics of the distributions are summarized in Table 1. Kolmogorov-Smirnov test results between estimates and reference data are near 0.1, indicating reasonable correspondence between the distributions [30]. As shown by Figure 3a, the positions of the peaks in the reference distribution are well reproduced by the $\mathrm{X}-\mu \mathrm{CT}$ estimate. The full width at half maximum of each peak in the $\mathrm{X}-\mu \mathrm{CT}$ estimate is on average $0.1(L / d)_{\text {peak }}$ higher than in the corresponding reference peak, where $(L / d)_{\text {peak }}$ denotes the position of the peak.

\section{Application to a composite manu- facturing process}

As a first practical application of the method discussed in Section 2, we study the degradation of fibres during manufacturing process of a wood fibre reinforced thermoplastic lignin composite. The material was manufactured from 20 wt-\% paper-grade softwood pulp (Metsä Fibre, Finland) and 80 wt-\% externally plasticised softwood kraft lignin (LignoBoost, Metso, Finland). The wood fibres and the externally plasticised lignin were compounded in a co-rotating twin-screw extruder (ZE $25 \times 48 \mathrm{D}$, Berstorff $\mathrm{GmbH}$, Germany) using a temperature profile of $40^{\circ} \mathrm{C}$ 
(feeding zone) $-160^{\circ} \mathrm{C}$ (die) and a specially designed screw geometry to provide even fibre dispersion. To facilitate feeding, the wood fibres were compacted into pellets using the sieve plate compression method described in [31]. After compounding, the extrudate was cooled down on a moving belt in air and cut into $5 \mathrm{~mm}$ granulates. ISO 3167 tensile test specimens ('dogbones') were manufactured using injection moulder with melt temperature of $160^{\circ} \mathrm{C}$ (ES 200/50 HL, Engel Austria GmbH). The injection pressure was 80 bar and the post pressure was 50 bar.

In order to study the changes in the structural characteristics of fibres appearing at various stages of the manufacturing process, samples of the original pulp, the pellets and the final composite were taken, imaged with XRadia $\mathrm{CCT}-400$ tomograph (XRadia, Concord, California, USA) and analysed using the method described above. The pulp and pellet samples were of size $2 \mathrm{~mm} \times 2 \mathrm{~mm} \times 4 \mathrm{~mm}$, and $2 \mathrm{~mm} \times 2 \mathrm{~mm} \times 2 \mathrm{~mm}$, respectively, and were imaged using $2.4 \mu \mathrm{m}$ resolution. The size of the composite material sample was approximately $1 \mathrm{~mm} \times 1 \mathrm{~mm} \times 1 \mathrm{~mm}$ and it was cut from the central part of the tensile test specimen with a rotary tool. The composite material sample was imaged with $1 \mu \mathrm{m}$ resolution. The reconstructed threedimensional images were bandpass filtered prior to processing, such that imaging noise and artefacts were suppressed. To separate fibres from air in the filtered image of original pulp fibres, the image was thresholded using Otsu method [32]. The filtered image of fibre pellets was thresholded similarly. For the image of the composite sample, the threshold value was chosen such that the volume fraction of objects identified as fibres coincided with the expected volume fraction determined from gravimetric composition and the densities of the constituents, namely 0.20 .

The aspect ratio of the fibres and the shape parameter $\left\langle d_{1}\right\rangle /\left\langle d_{2}\right\rangle$ of the fibre cross-sections were determined from 50000 cross-sectional samples, as described in Section 2. Based on the results shown in Figure 4, it is apparent that the aspect ratio decreases considerably as the pulp fibres are processed into pellets and further into injection moulded dogbone specimen. The value of the shape parameter $\left\langle d_{1}\right\rangle /\left\langle d_{2}\right\rangle$ differs from unity, indicating non-circular shape of fibre cross-sections.

\section{Application to micromechanical modelling}

We next apply the method discussed in Section 2 in finding structural parameters needed in the rule-of-mixtures model presented by Madsen et. al. [33] for estimating stiffness of composite materials. To this end, four types of flax fibre/starch acetate composites, labeled C1, C2, C3 and C4, were manufactured (see Table 2). Flax fibres and amylose rich corn starch were supplied by Ekotex (Poland) and Gargill (USA), respectively. The manufacturing process, including fibre pelletizing, starch acetylation and plasticization, is as described in [34] with the exception that, in the present case, all compounds and composites were made with temperature gradient from $60{ }^{\circ} \mathrm{C}$ in the compounder feeding section to $200{ }^{\circ} \mathrm{C}$ in the melting zones and die. In the process the original flax fibres tend to become partially fragmented such that the final composite may also contain smaller segments and pieces of fibres ('fines') with a wide distribution of size and shape. The experimental values of Young's modulus of the materials were determined by tensile tests according to ISO 527 standard $[2,34]$.

According to the rule-of-mixtures model [33] the Young's modulus $E_{c}$ of a composite material is given by

$$
E_{c}=\left(\eta_{o} \eta_{l} V_{f} E_{f}+V_{m} E_{m}\right)\left(1-V_{v}\right)^{2},
$$

where $V_{f}, V_{m}$ and $V_{v}$ are the volume fractions of fibres, matrix and void, respectively, and $\eta_{o}$ and $\eta_{l}$ are the orientation and the length efficiency factors, respectively.

The orientation efficiency factor is given by [1]

$$
\eta_{o}=\sum_{n} a_{n} \cos ^{4}\left(\alpha_{n}\right)
$$

where $\alpha_{n}$ is the angle between the loading direction and the direction of $n$ :th fibre and $a_{n}=V_{f n} / V_{f}$, where $V_{f n}$ is the volume fraction of $n$ :th fibre. The length efficiency factor is given by [35]

$$
\eta_{l}=1-\frac{\tanh \left(L^{*}\right)}{L^{*}}
$$

where the shear lag parameter $L^{*}$ is given by

$$
L^{*}=2 \frac{\langle L\rangle}{\langle d\rangle} \sqrt{\frac{G_{m}}{E_{f} \ln \left(\kappa / V_{f}\right)}} .
$$


Here, the angle brackets denote mean value over individual fibres, and $E_{f}, E_{m}$ and $G_{m}$ are the Young's modulus of fibres, the Young's modulus of matrix and the shear modulus of the matrix, respectively. The latter is given by $G_{m}=E_{m} / 2\left(1+\nu_{m}\right)$ assuming isotropic material with Poisson's ratio $\nu_{m}=0.3 \pm 0.05$. The fibres are assumed to be packed hexagonally, implying the value of the geometrical packing pattern constant $\kappa=\frac{\pi}{2 \sqrt{3}} \approx 0.907$. The value of Young's modulus of the fibres is taken to be $E_{f}=50 \mathrm{GPa} \pm 5 \mathrm{GPa}$ according to the results by Lilholt and Lawther [36]. The values of the remaining material dependent parameters are given in Table 2.

The yet unknown parameters of the model, namely the aspect ratio $\langle L\rangle /\langle d\rangle$ and the orientation efficiency factor $\eta_{o}$ were estimated using the data obtained from $\mathrm{X}-\mu \mathrm{CT}$ images of material samples as discussed in Section 2. For $\mathrm{X}-\mu \mathrm{CT}$ analysis, a cylindrical subsample of diameter $2 \mathrm{~mm}$ was cut from the central region of each injection moulded tensile test specimen using a rotary tool, and attached on

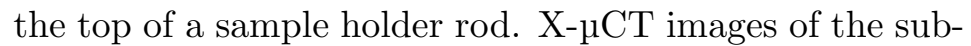
samples were taken using XRadia $\mu \mathrm{CT}-400$ device (XRadia, Concord, California, USA) with voxel size of $1.24 \mu \mathrm{m}$ and cylindrical image volume of diameter $2 \mathrm{~mm}$ and height $2 \mathrm{~mm}$. The images were denoised using variance weighted mean filter and binarized to segment the flax particles from the matrix. The threshold gray-scale value was selected such that the volume fraction of the reinforcement phase coincided with the result from gravimetric measurements [34]. The result of the filtering and segmentation operations is visualized in Figure 5 . The length $L_{i}$, the principal dimension $d_{1 i}$ and the angle $\alpha_{i}$ between the normal direction and loading direction were determined for 50000 randomly selected samples of particle cross-section as described in Section 2.

The rule-of-mixtures model assumes the reinforcement phase to consist of fibres long and thin enough such that the load is transferred between the matrix and a fibre only in the direction of the fibre [35]. This assumption gives rise to orientation and length efficiency factors appearing in Equation (4). In the present case, the materials may also contain very short fibre fragments with low aspect ratio which are not compliant with that assumption and which, on the other hand, can be considered less effective in reinforcing the composite. Such particles should thus be excluded from calculation of the values of $\eta_{o}$ and $\eta_{l}$. To this end, we first calculate the shear lag parameter $L_{i}^{*}$ for each individual particle cross section $i$ according to Equation (7), but replacing $\langle L\rangle /\langle d\rangle$ by $L_{i} / d_{1 i}$. The quantity $L_{i}^{*}$ can be interpreted as a dimensionless effective fibre length and a natural cut-off is obtained by excluding particles with $L_{i}^{*}<1$. For the present composites this corresponds to excluding particles with aspect ratio $L_{i} / d_{1 i}$ less than $\sim$ 5-10. The total amount of cross-sectional samples thereby discarded varied between $20 \%$ and $55 \%$ by volume, depending on the material. The orientation efficiency factor $\eta_{o}$, length efficiency factor $\eta_{l}$, and Young's modulus $E_{c}$ were then calculated for each material based on the remaining set of cross-sections associated with elongated particles ('fibres') using Equations (4) through (7). Another possibility for excluding small fibre fragments within the present method would be to introduce a cut-off value $L_{c}^{*}$ as a free parameter and find its value by fitting the predicted values of $E_{c}$ to experimental results. For the present set of data such fitting yields $L_{c}^{*}=0.90 \pm 0.08$ and leads to estimates on $E_{c}$ practically identical with those obtained with the choice $L_{c}^{*}=1$ made above based solely on dimensional arguments (see below).

Notice that in the random sampling process used in the present method, the probability of sampling a crosssection of $n$ :th fibre is equal to $V_{f n} / V_{f}=a_{n}$. Consequently, the average over individual fibres of variable $x=\{L, d\}$ is given by

$$
\langle x\rangle=\frac{\sum_{i=1}^{N} \frac{x_{i}}{L_{i} A_{i}}}{\sum_{i=1}^{N} \frac{1}{L_{i} A_{i}}},
$$

and the orientation efficiency factor $\eta_{o}$, Equation (5), by

$$
\eta_{o}=\frac{1}{N} \sum_{i=1}^{N} \cos ^{4}\left(\alpha_{i}\right),
$$

where the sums are taken over the $N$ randomly selected cross-sections associated with fibres, i.e. particles with $L_{i}^{*} \geq 1$.

For comparison, the model parameters were estimated also using a manual reference method based on first dissolving the matrix in hot chloroform and collecting the remaining material. Microscopic images of the remaining material were taken, and the length and the width of fibres were measured manually [37]. Small fragments of fibre were discarded as 'fines' based on visual inspection (corresponding to the exclusion of low aspect ratio particles based on $L_{i}^{*}$ cut-off in the method discussed above). 
The value of $\langle L\rangle /\langle d\rangle$ was then calculated and substituted into Equations 4-7. The value of the orientation efficiency factor $\eta_{o}$ could not be determined within the reference method, and was found by fitting the Young's moduli given by Equation 4 to the results of the mechanical tests using $\eta_{o}$ as a free parameter. The optimal value thereby found was $\eta_{o} \approx 0.66 \pm 0.05$, which was used for all four materials.

The properties of the composites as estimated using the $\mathrm{X}-\mu \mathrm{CT}$ method and the reference method are shown in Table 2. The results on $\langle L\rangle /\langle d\rangle$ given by the two methods are similar but not identical. A possible reason for the difference is the dissimilar algorithm used in discarding fines within the two methods. In addition, during the manual measurement, fibres may lie on the microscope slide in random orientations. This is likely to lead in diameter estimates that do not directly correspond to the major cross-sectional principal dimension $d_{1}$ used within the $\mathrm{X}-\mu \mathrm{CT}$ approach.

The various estimates of Young's modulus are shown in Figure 6, together with values from mechanical tests. Both the $\mathrm{X}-\mu \mathrm{CT}$ method and the reference method give estimates similar to the results from the mechanical tests. In all the cases, the estimates obtained by the $\mathrm{X}-\mu \mathrm{CT}$ method agree with the experimental results within error limits.

As mentioned above, a substantial fraction of all particles found in the $\mathrm{X}-\mu \mathrm{CT}$ images of the material samples studied here were discarded as small fibre fragments. This was done merely in order to comply with the assumptions of the rule-of-mixture model used and to mimic the procedures inherent in the manual reference method discussed in this demonstrative example. Obviously, the detailed information available from the $\mathrm{X}-\mu \mathrm{CT}$ data could be used also with more advanced micromechanical models in obtaining the necessary statistical properties including all observable particles, irrespective of their size.

\section{Discussion}

A non-destructive, fully automated method for estimating the length, aspect ratio and cross-sectional properties of reinforcement fibres in a composite material was introduced. The method is based on analysing X-ray microtomographic images of material samples. It requires segmentation of the fibre phase from the matrix material in the three-dimensional tomographic images, but it does not require identification of individual fibres. It can differentiate various types of fibres, e.g. solid and hollow fibres, based on their cross-sectional geometry. The method also helps reducing biasing that may be present in conventional methods based on, e.g., dissolution of matrix material, visual identification of fibres with certain properties, and manual counting.

The most important limitations of the method are related to finite tomographic image size, resolution and contrast available. However, assessment of feasibility of the method as well as quantitative error analysis can be validly done only in relation to each particular application. Some general guidelines towards such assessment can, nevertheless, be given here. The basic prerequisites for applicability of the method for a particular composite material are that the density difference between the fibres and the matrix is large enough such that they can be adequately segmented in the tomographic image, and that the size of the smallest details discernible in tomographic images (the detail detectability) is small as compared to the smallest fibre dimension, preferably by an order of magnitude. Furthermore, the typical fibre length should not exceed the smallest physical dimension of the tomographic image (field of view within the scanner set-up used). Even if this is the case, the the finite tomographic image size can cause biasing in analysis of especially fibre length. The error caused by such biasing can, however, be reduced by methods such as that introduced by Miettinen et al. [25]. The method is thus potentially useful also in cases where a fraction of fibres are longer than the image dimension. Obtaining statistically relevant results for heterogeneous materials with the size scale of heterogeneities larger than the physical dimensions of the tomographic image may require using an increased number of samples, or particular care in selecting samples representative of the material. The uncertainty of the results is also likely to increase with fibre content and with increased complexity of fibre shape.

As the first example of application of the method, we assess the degree of morphological degradation of fibres in various stages of manufacturing process of a wood fibre reinforced thermoplastic lignin composite. The process included compaction of the initial pulp into pellets, compounding with plasticised lignin and injection moulding. The results show total of $50 \%$ decrease in fibre aspect ra- 
tio, a major part of which occurred prior to compounding.

Secondly, the method was demonstrated in estimating stiffness of a set of flax fibre/starch acetate composites using a micromechanical rule-of-mixtures model. Here, the values of crucial model parameters, namely the volume fraction of fibres and of voids, the mean fibre aspect ratio and the fibre orientation efficiency factor were found utilising the present method. The results agree well with the experimental values of Young's modulus.

\section{Acknowledgements}

The authors acknowledge high-school students Solja AlaKorpi and Riikka Hirvelä for valuable laboratory work in manufacturing the model composite during their interim period at the University of Jyväskylä, Department of Physics (June 2013). This project has been partially funded through Finnish Bioeconomy Cluster (FIBIC) FuBio Joint Research 2 program.

\section{References}

[1] Krenchel H. Fibre reinforcement. Ph.D. thesis; Copenhagen; 1964.

[2] Madsen B, Joffe R, Peltola H, Nättinen K. Short cellulosic fiber/starch acetate composites - micromechanical modeling of Young's modulus. Journal of Composite Materials 2011;45(20):2119-2131.

[3] Neagu C, Gamstedt E, Berthold F. Stiffness contribution of various wood fibers to composite materials. Journal of Composite Materials 2006;40:663-699.

[4] Andersons J, Joffe R, Spārniņš E. Stiffness and strength of flax fiber/polymer matrix composites. Polymer Composites 2006;27:221-229.

[5] Nyström B. Natural fiber composites - optimization of microstructure and processing parameters. Licentiate thesis; Luleå University of Technology, Department of Applied Physics and Mechanical Engineering; 2007.

[6] Gamstedt EK, Joffre T, Miettinen A, Berthold F. Monitoring of fibre length degradation during processing of short-fibre composites by use of X-ray computed tomography. In: Proceedings of the 34th Ris $\varnothing$
International Symposium on Materials Science. Department of Wind Energy, Risø Campus, Technical University of Denmark; 2013,.

[7] Joffre T, Miettinen A, Berthold F, Gamstedt EK. Xray micro-computed tomography investigation of fibre length degradation during the processing steps of short-fibre composites; 2014. In preparation.

[8] Ho M, Wang H, Lee J, Ho C, Lau K, Leng J, et al. Critical factors on manufacturing processes of natural fibre composites. Composites Part B: Engineering 2012;43(8):3549 - 3562 .

[9] Barkoula NM, Garkhail SK, Peijs T. Effect of compounding and injection molding on the mechanical properties of flax fiber polypropylene composites. Journal of reinforced plastics and composites 2010;29(9).

[10] El-Sabbagh AMM, Steuernagel L, Meiners D, Ziegmann G. Effect of extruder elements on fiber dimensions and mechanical properties of bast natural fiber polypropylene composites. Journal of Applied Polymer Science 2014;131(12).

[11] Kak AC, Slaney M. Principles of Computerized Tomographic Imaging. IEEE Press; 1988.

[12] Gonzalez R, Woods R. Digital Image Processing. Prentice-Hall; 2 ed.; 2002.

[13] Clarke A, Eberhardt C. The representation of reinforcing fibres in composites as 3D space curves. Composites Science and Technology 1999;59(8):1227 $-1237$.

[14] Yang H, Lindquist WB. Three-dimensional image analysis of fibrous materials. In: Proceedings of SPIE; vol. 4115. 2000, p. 275-282.

[15] Tan J, Elliott J, Clyne T. Analysis of tomography images of bonded fibre networks to measure distributions of fibre segment length and fibre orientation. Advanced Engineering Materials 2006;8(6):495-500.

[16] Lux J, Delisée C, Thibault X. 3D characterization of wood based fibrous materials: an application. Image Analysis \& Stereology 2011;25(1). 
[17] Tsarouchas D, Markaki A. Extraction of fibre network architecture by X-ray tomography and prediction of elastic properties using an affine analytical model. Acta Materialia 2011;59(18):6989-7002.

[18] Walther T, Terzic K, Donath T, Meine H, Beckmann F, Thoemen H. Microstructural analysis of lignocellulosic fiber networks. In: Proceedings of SPIE; vol. 6318. 2006, p. 631812-631812-10.

[19] Axelsson M. 3D tracking of cellulose fibres in volume images. In: Image Processing, 2007. ICIP 2007. IEEE International Conference on; vol. 4. 2007, p. IV - 309IV -312 .

[20] Wernersson E, Brun A, Luengo Hendriks C. Segmentation of wood fibres in 3D CT images using graph cuts. In: Foggia P, Sansone C, Vento M, editors. Image Analysis and Processing - ICIAP 2009; vol. 5716 of Lecture Notes in Computer Science. Springer Berlin Heidelberg; 2009, p. 92-102.

[21] Malmberg F, Lindblad J, Östlund C, Almgren K, Gamstedt E. Measurement of fibre-fibre contact in three-dimensional images of fibrous materials obtained from X-ray synchrotron microtomography. Nuclear Instruments and Methods in Physics Research Section A: Accelerators, Spectrometers, Detectors and Associated Equipment 2011;637(1):143-148.

[22] Eberhardt CN, Clarke AR. Automated reconstruction of curvilinear fibres from 3D datasets acquired by X-ray microtomography. Journal of Microscopy 2002;206(1):41-53.

[23] Teßmann M, Mohr S, Gayetskyy S, Haßler U, Hanke R, Greiner G. Automatic determination of fiberlength distribution in composite material using $3 \mathrm{D}$ CT data. EURASIP Journal of Advanced Signal Processing 2010;2010.

[24] Viguié J, Latil P, Orgéas L, Dumont P, Rolland du Roscoat S, Bloch JF, et al. Finding fibres and their contacts within 3D images of disordered fibrous media. Composites Science and Technology 2013;89:202 -210 .

[25] Miettinen A, Luengo Hendriks CL, Chinga-Carrasco G, Gamstedt EK, Kataja M. A non-destructive Xray microtomography approach for measuring fibre length in short-fibre composites. Composites Science and Technology 2012;72:1901-1908.

[26] Miettinen A, Kataja K. Non-destructive analysis of fiber properties using 3D X-ray microtomographic data. In: Proceedings of the 32nd Ris $\varnothing$ International Symposium on Materials Science. Department of Wind Energy, Risø Campus, Technical University of Denmark; 2011,.

[27] Jähne B. Practical Handbook on image processing for scientific and technical applications. CRC Press; 2004 .

[28] Pearson K. On lines and planes of closest fit to systems of points in space. Philosophical Magazine 1901;2:559-572.

[29] Luengo Hendriks CL. Constrained and dimensionality-independent path openings. IEEE Transactions on Image Processing 2010;19(6):15871595.

[30] A. K. Sulla determinazione empirica di una legge di distribuzione. Giornale dell'Istituto Italiano degli Attuari 1933;4:83-91.

[31] Sivonen E, Valta K. Seulalevypuristin. Finnish patent application FI20106340; 2012.

[32] Otsu N. A threshold selection method from gray level histograms. IEEE Transactions on Systems, Man and Cybernetics 1979;9:62-66.

[33] Madsen B, Thygesen A, Lilholt H. Plant fibre composites - porosity and stiffness. Composites Science and Technology 2009;69(7-8):1057-1069.

[34] Nättinen K, Hyvärinen S, Joffe R, Wallström L, Madsen B. Naturally compatible: Starch acetate/cellulosic fiber composites. I. processing and properties. Polymer Composites 2010;31(3):524-535.

[35] Cox H. The elasticity and strength of paper and other fibrous materials. British Journal of Applied Physics 1952;3:72-79.

[36] Lilholt H, Lawther J. Natural organic fibres. In: Kelly A, Zweben C, editors. Comprehensive composite materials; vol. 1; chap. 10. Amsterdam: Elsevier Science; 2000, p. 303-325. 
[37] Peltola H, Madsen B, Joffe R, Nättinen K. Experimental study of fiber length and orientation in injection molded natural fiber/starch acetate composites. Advances in materials science and engineering $2011 ; 2011$ 


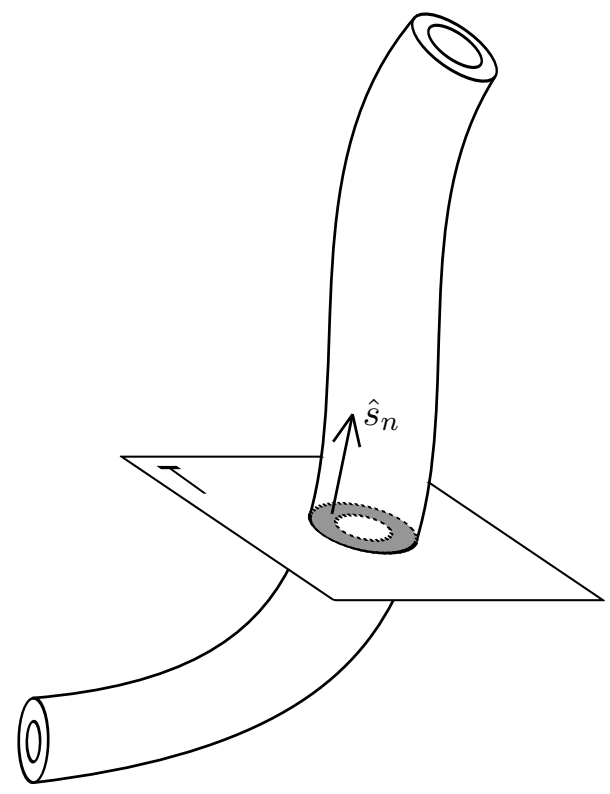

(a)

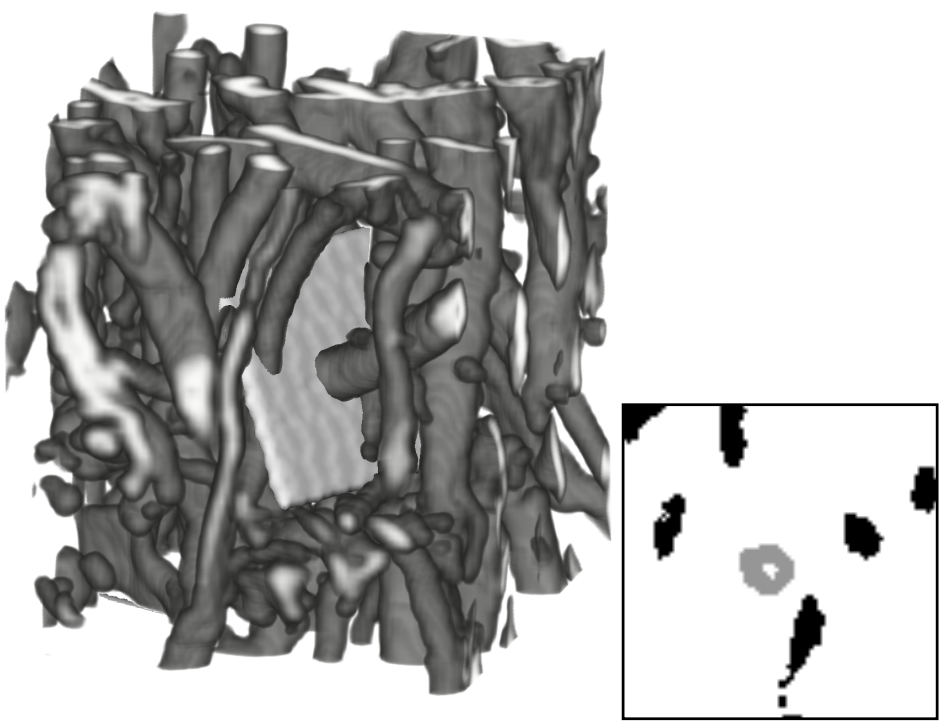

(b)

Figure 1: (a) Slicing a single fibre. Slice $\mathrm{T}$ is taken such that its normal $\hat{s}_{n}$ is parallel with the local fibre orientation

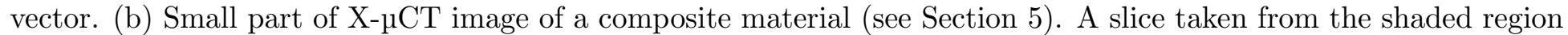
is shown in the inset.

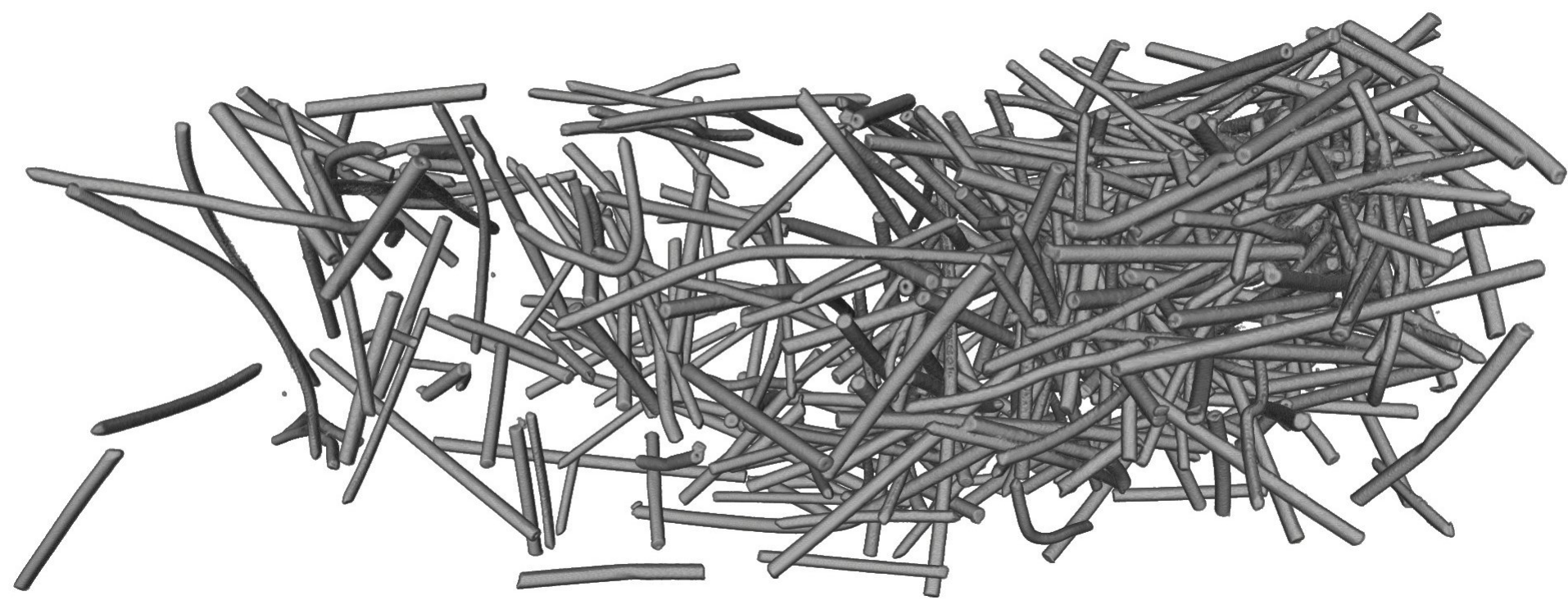

Figure 2: Three-dimensional visualization of the metallic fibres in the model composite. The width of the sample is approximately $5 \mathrm{~cm}$. The rubber matrix is not shown. 


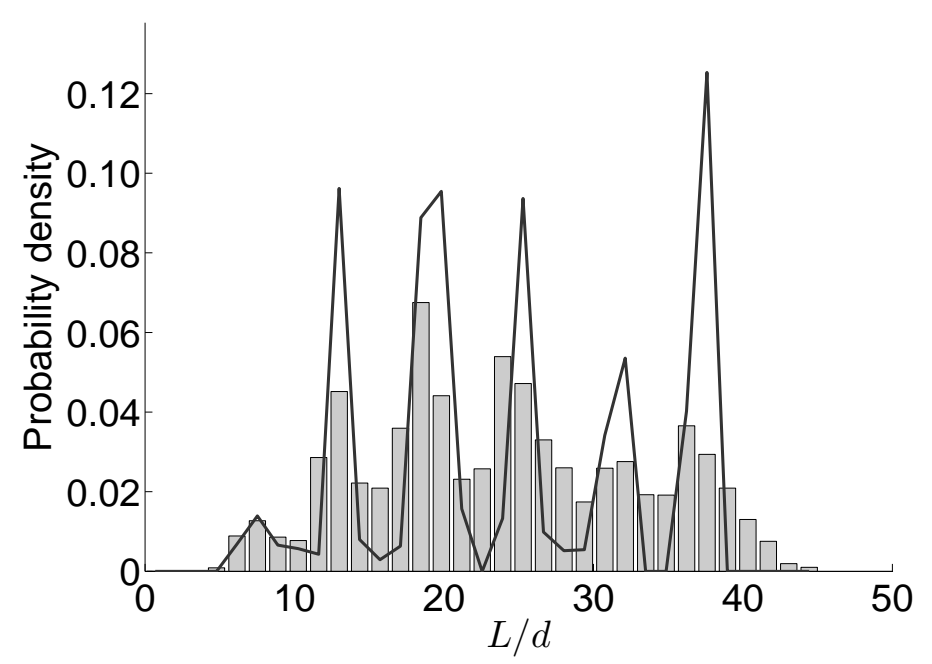

(a)

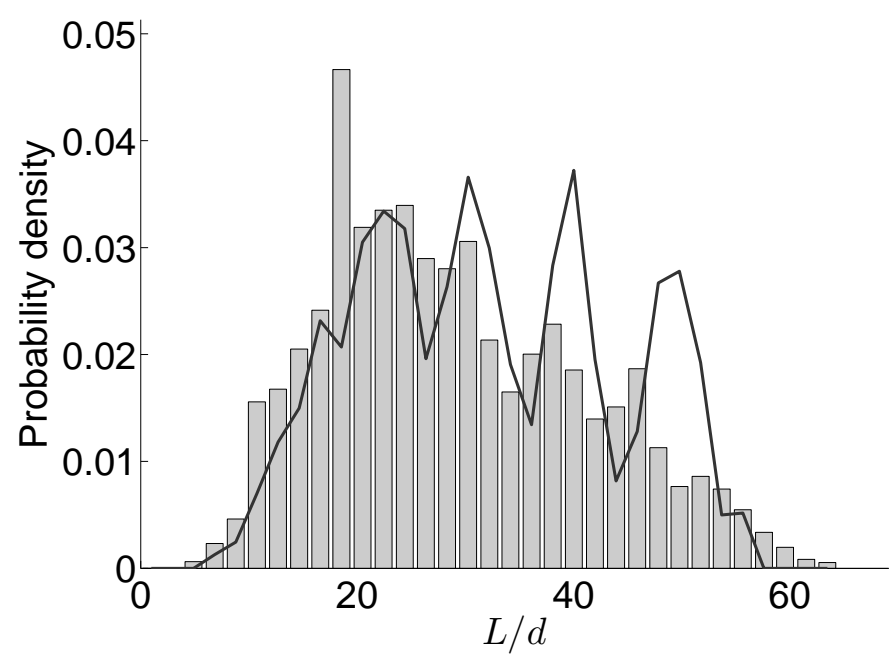

(b)

Figure 3: Aspect ratio distribution for (a) hollow fibres and (b) solid fibres in the model composite. The bar plot is the distribution as estimated by the $\mathrm{X}-\mathrm{\mu CT}$ method and the solid line is the reference distribution obtained from the independent manual counting method.

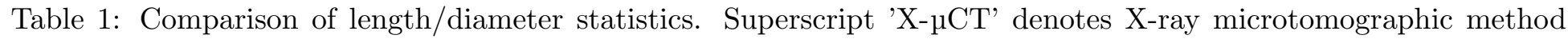
whereas superscript 'ref' denotes the reference method. Angle brackets denote mean, $\sigma$ denotes standard deviation, $\Delta$ denotes relative difference between the two means and K-S denotes the result of Kolmogorov-Smirnov test [30]. All the quantities are dimensionless.

\begin{tabular}{ccccccc} 
Fibre type & $\langle L / d\rangle^{\mathrm{X}-\mu \mathrm{CT}}$ & $\langle L / d\rangle^{\mathrm{ref}}$ & $\sigma^{\mathrm{X}-\mu \mathrm{CT}}$ & $\sigma^{\text {ref }}$ & $\Delta / \%$ & $\mathrm{~K}-\mathrm{S}$ \\
\hline Hollow & 23.9 & 24.5 & 9.0 & 9.1 & 2.3 & 0.076 \\
Solid & 29.0 & 32.1 & 12.0 & 11.8 & 9.4 & 0.13
\end{tabular}

Table 2: Properties of the flax fibre/starch acetate composites. $V_{f}, V_{m}$ and $V_{v}$ are volume fractions of fibres, matrix and void, respectively. $P$ is the plasticizer content in the matrix, $E_{m}$ is the Young's modulus of the matrix, $G_{m}$ is the shear modulus of the matrix, and $\eta_{o}$ is the orientation efficiency factor. Superscript 'X- $\mu \mathrm{CT}$ ' refers to the X- $\mu \mathrm{CT}$ method and 'ref' to the reference method. Orientation efficiency factor $\eta_{o}^{\text {ref }} \approx 0.66 \pm 0.05$. The uncertainties of aspect ratios are obtained using error propagation law with the standard deviations of the mean of length and diameter. The uncertainties of volume fractions and orientation efficiency factors are taken to be 0.01.

\begin{tabular}{cccccccccc} 
Sample & $V_{f}$ & $V_{m}$ & $V_{v}$ & $P[\mathrm{wt}-\%]$ & $E_{m}[\mathrm{GPa}]$ & $G_{m}[\mathrm{GPa}]$ & $\langle L\rangle /\left\langle d_{1}\right\rangle^{\mathrm{X}-\mu \mathrm{CT}}$ & $\langle L\rangle /\langle d\rangle^{\mathrm{ref}}$ & $\eta_{o}^{\mathrm{X}-\mu \mathrm{CT}}$ \\
\hline $\mathrm{C} 1$ & 0.11 & 0.89 & 0.00 & 20.0 & $1.66 \pm 0.05$ & $0.64 \pm 0.04$ & $13.4 \pm 0.1$ & $19.3 \pm 0.1$ & 0.61 \\
$\mathrm{C} 2$ & 0.36 & 0.62 & 0.01 & 20.0 & $1.66 \pm 0.05$ & $0.64 \pm 0.04$ & $10.6 \pm 0.1$ & $7.9 \pm 0.1$ & 0.64 \\
$\mathrm{C} 3$ & 0.09 & 0.91 & 0.00 & 32.5 & $0.45 \pm 0.05$ & $0.17 \pm 0.03$ & $26.3 \pm 0.3$ & $17.6 \pm 0.1$ & 0.61 \\
$\mathrm{C} 4$ & 0.36 & 0.64 & 0.01 & 32.5 & $0.45 \pm 0.05$ & $0.17 \pm 0.03$ & $19.1 \pm 0.2$ & $15.4 \pm 0.1$ & 0.54
\end{tabular}




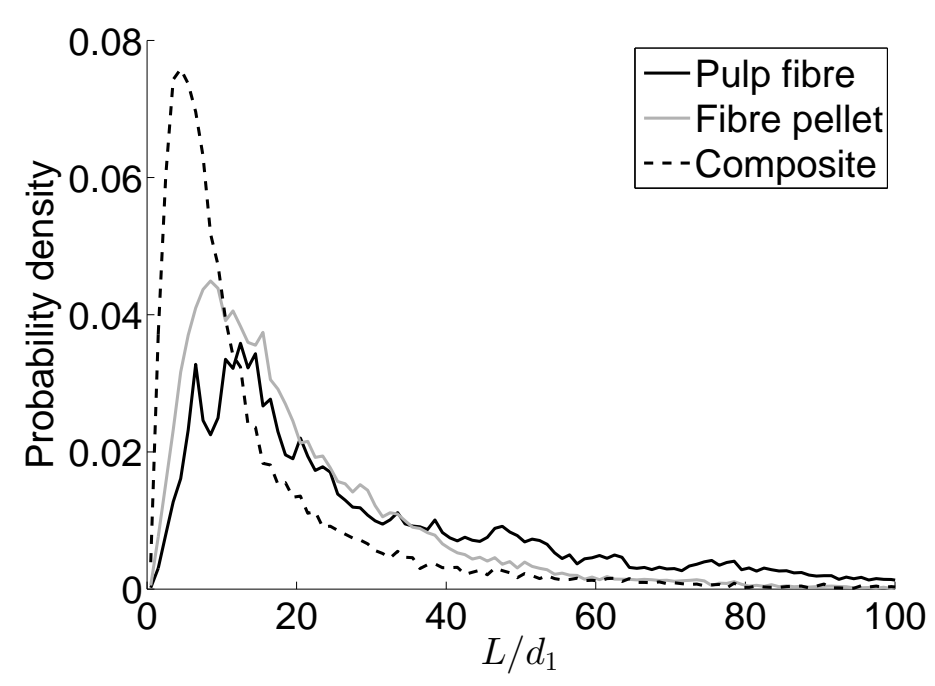

(a)



(c)

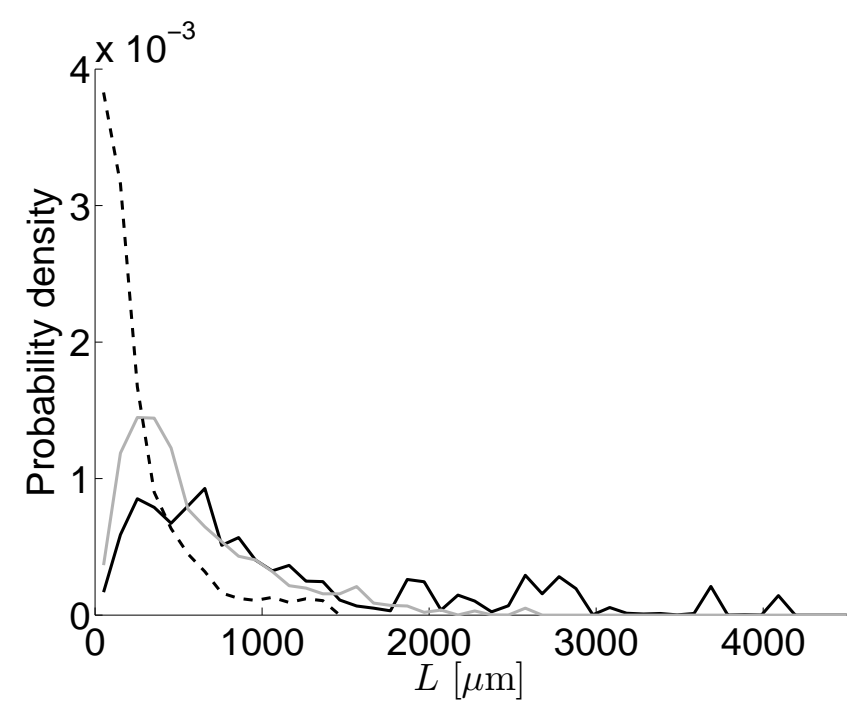

(b)

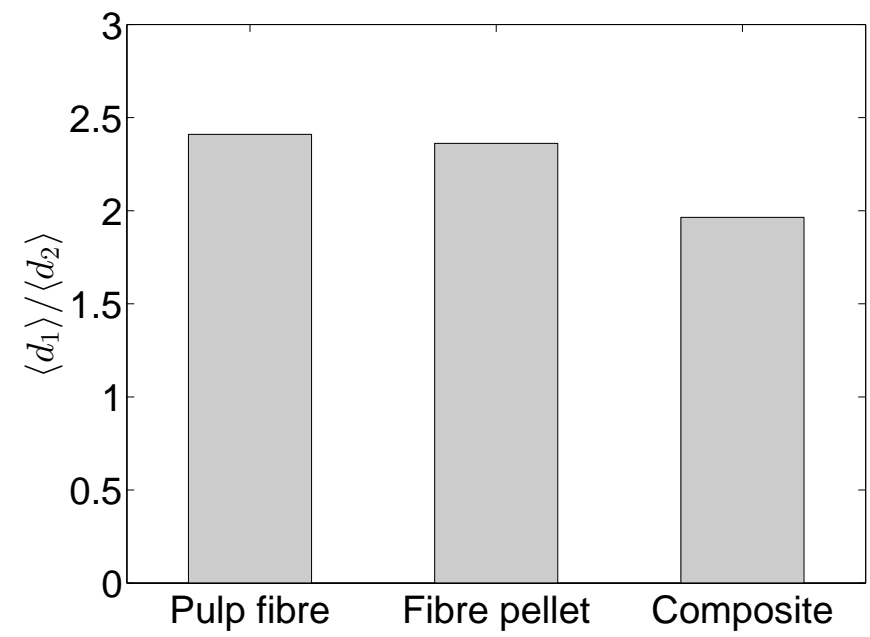

(d)

Figure 4: (a) Aspect ratio distributions, (b) length distributions, (c) aspect ratios, and (d) cross-sectional shape parameter $\left\langle d_{1}\right\rangle /\left\langle d_{2}\right\rangle$ at various stages of composite manufacturing process. The distributions shown in (a) and the mean values given in (c) are determined based on the major cross-sectional diameter $d_{1}$. 


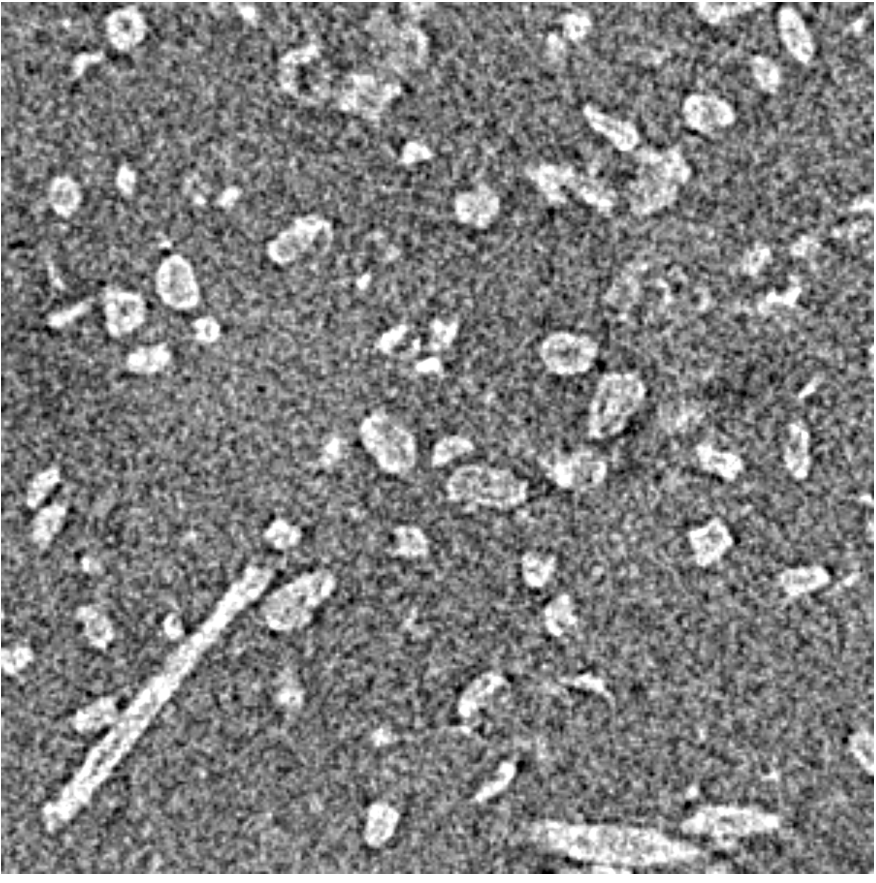

(a)

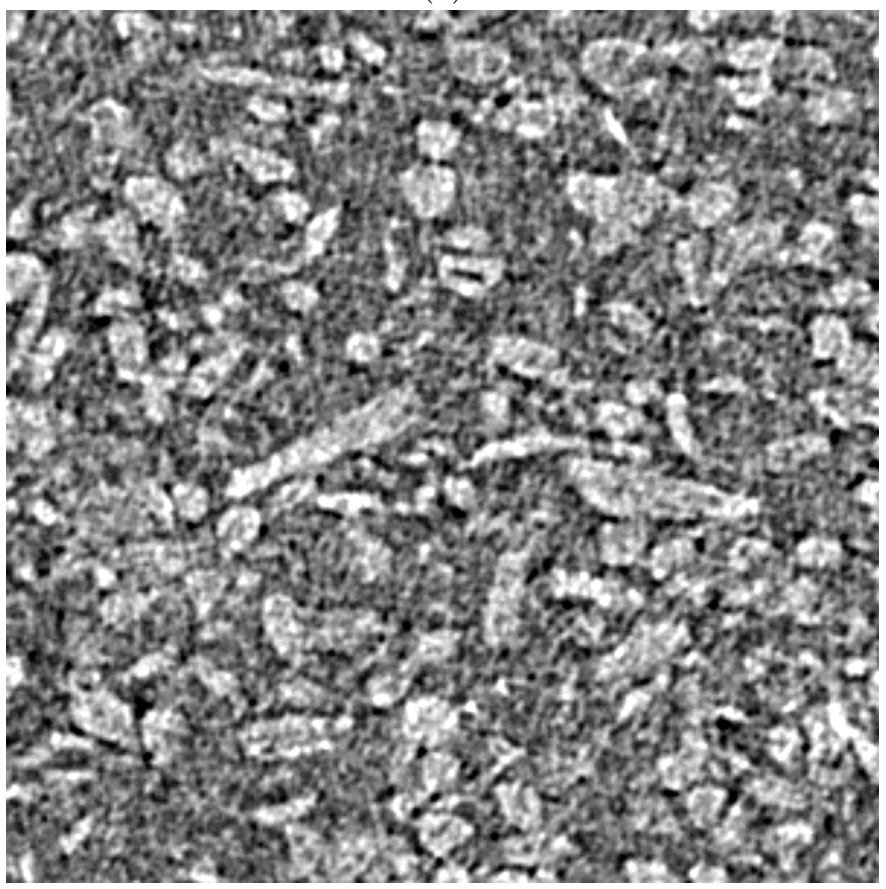

(c)

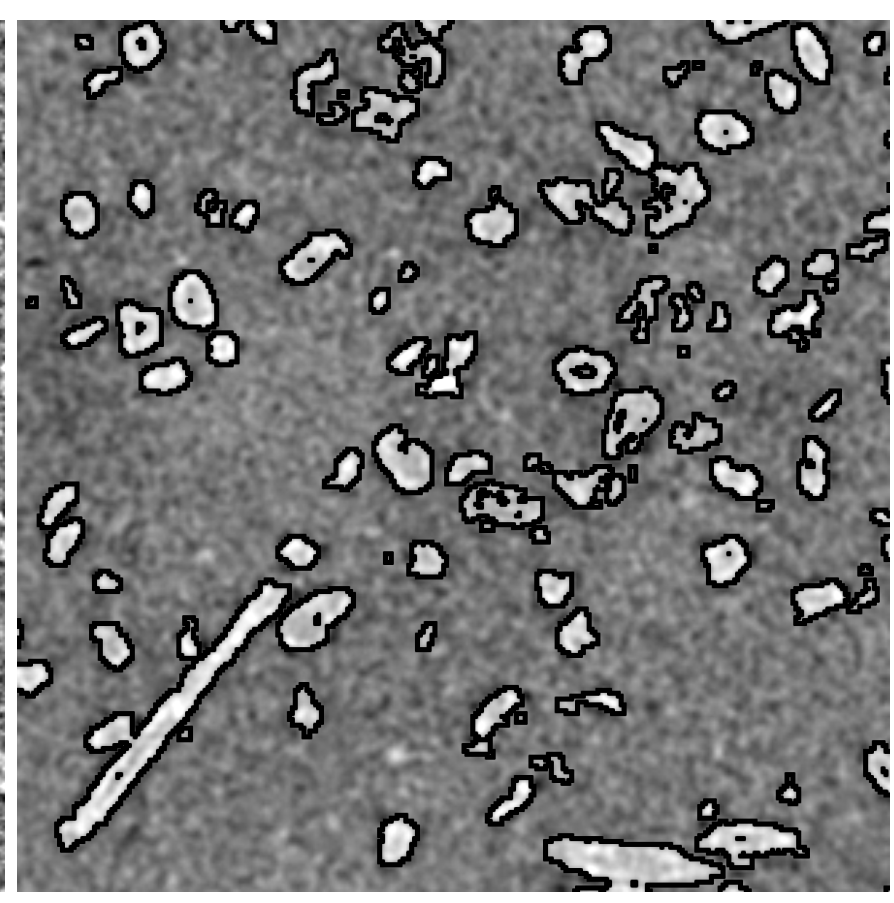

(b)

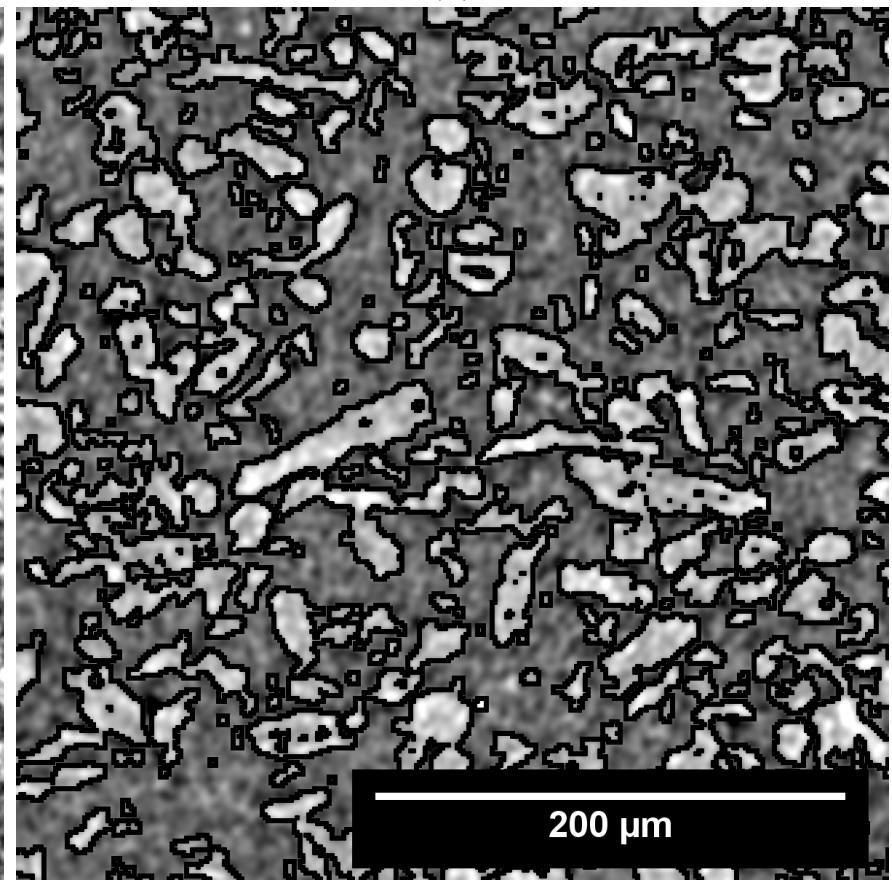

(d)

Figure 5: (a) A small part of a single unfiltered slice of $\mathrm{X}-\mu \mathrm{CT}$ image of sample C1. Fibre cross-sections and matrix are shown as light gray and varying darker gray regions, respectively. (b) The image in (a) after variance weighted mean filtering and segmenting. The boundary between segmented fibre wall and matrix is shown as black line. (c), (d) Same as (a) and (b) but for sample C4. 


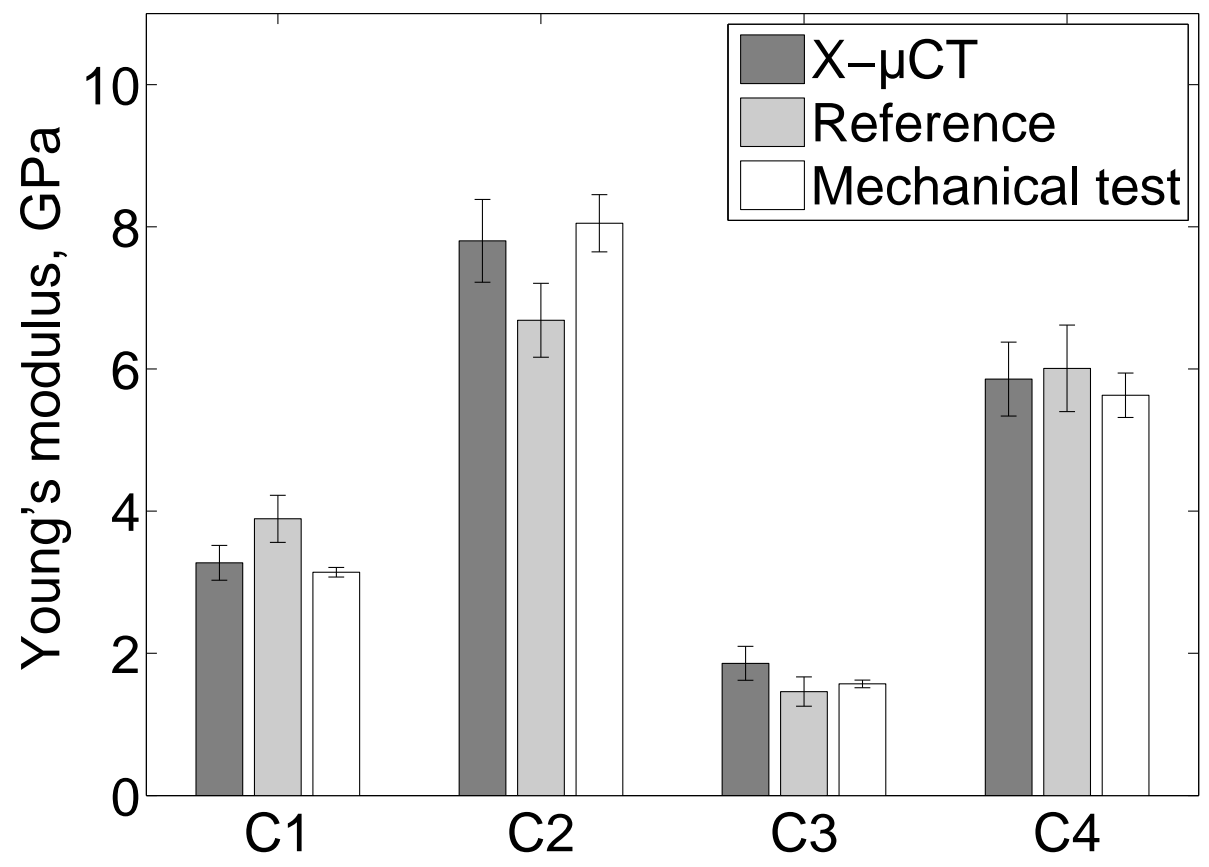

Figure 6: Estimates of Young's modulus of the flax fibre composites determined using Equation 4 with parameters estimated using the $\mathrm{X}-\mu \mathrm{CT}$ method and the manual reference method. Also given are the experimental results from mechanical tests. The error limits for the estimates are obtained using the error propagation law and the error limits given for various parameters. The error limits of the experimental results are standard errors of the mean over five measured samples. 